\title{
The Potential of Multi-Junction Perovskite Solar Cells
}

\author{
Maximilian T. Hörantner ${ }^{1,4 \ddagger^{*}}$, Tomas Leijtens ${ }^{2 \ddagger}$, Mark E. Ziffer ${ }^{3}$, Giles E. Eperon ${ }^{3,5}$, M. \\ Greyson Christoforo ${ }^{4}$, Michael D. McGehee ${ }^{2}$, Henry J. Snaith ${ }^{4 *}$ \\ ${ }^{1}$ Department of Electrical Engineering and Computer Science, Massachusetts Institute of \\ Technology, 77 Massachusetts Ave., Cambridge, USA \\ ${ }^{2}$ Department of Materials Science, Stanford University, 476 Lomita Mall, Stanford CA \\ 94305, United States \\ ${ }^{3}$ Department of Chemistry, University of Washington, Seattle, Washington WA 98195, USA \\ ${ }^{4}$ Department of Physics, University of Oxford, Clarendon Laboratory, Parks Road, OX1 3PU, \\ Oxford, United Kingdom \\ ${ }^{5}$ Cavendish Laboratory, JJ Thomson Avenue, Cambridge CB3 OHE, UK \\ These authors contributed equally to the work. \\ *Corresponding authors: maxhoer@mit.edu, henry.snaith@physics.ox.ac.uk
}

\begin{abstract}
Metal halide perovskite semiconductors offer rapid, low cost deposition of solar cell active layers with a wide range of bandgaps, making them ideal candidates for multi-junction solar cells. Here, we combine optical and electrical models using experimental inputs to evaluate the feasible performances of all perovskite double- (2PJ), triple-junction (3PJ), and perovskiteperovskite-silicon triple junction (2PSJ) solar cells. Using parameters and design constraints from the current state of the art generation of perovskite solar cells, we find that 2PJs can feasibly approach $32 \%$ power conversion efficiency, 3PJs can reach $33 \%$, and 2PSJs can surpass $35 \%$. We also outline pathways to improve light harvesting and demonstrate that it is
\end{abstract}


possible to raise the performances to $34 \%, 37 \%$ and $39 \%$ for the three architectures. Additionally, we discuss important future directions of research. Finally, we perform energy yield modelling to demonstrate that the multijunction solar cells should not suffer from reduced operational performances due to discrepancies between the AM1.5G and real-world spectrum over the course of a year.

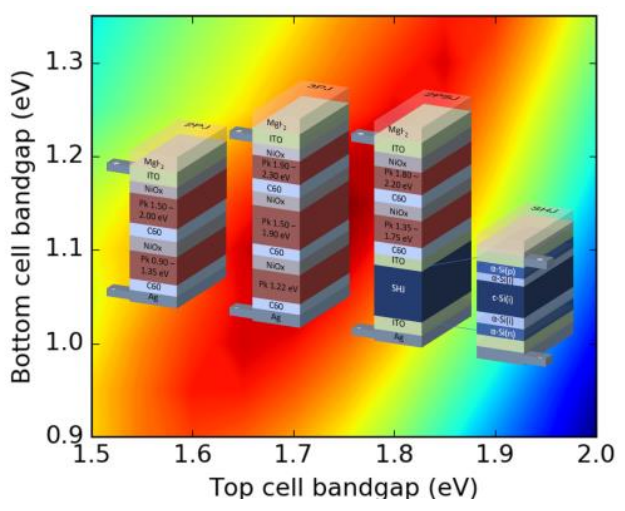

Metal halide perovskite solar cells are rapidly approaching performances that can rival those of crystalline silicon (c-Si). After only 5 years of intensive research, the record certified perovskite research solar cell efficiency is $22.1 \%^{1}$, while the record certified multi-crystalline silicon cells are at $21.9 \%$, which is the dominant commercially deployed PV technology ${ }^{2}$. For the most advanced c-Si concepts, which arise from lab based research, the last efficiency gains are being squeezed out, with efficiencies approaching $27 \%{ }^{3}$. Although improvements in perovskite solar cell efficiency can be expected over the next few years, single junction perovskite solar cells will always be limited to performances similar to those of c-Si. Mainstream PV module manufacturing costs have continued to diminish so extensively over the last decade that now the cost of the module amounts to less than half the overall solar PV installation in utility scale projects. Most of the non-module costs, referred to as the balance of systems (BoS), scale with area of deployed PV rather than power generated. Therefore, increasing the overall power output of the module per unit area, i.e. efficiency, is the surest means to continue to drive down the overall cost of installed PV generated electricity. 
Therefore, we need to develop a strategy and road map, which will lead perovskite solar cells to much higher efficiency than c-Si.

Multijunction solar cells using lattice matched III-V compound semiconductors such as GaAs and GaInP have thus far achieved the highest performances, reaching $31.1 \%$ in double (“tandem") junction, and obtaining $37.9 \%$, and $38.8 \%$ for triple and quadruple junction solar cells respectively, measured under standard simulated air-mass (AM) 1.5 solar irradiance at $100 \mathrm{mWcm}^{-2}{ }^{2}$ Unfortunately however, the metal organic chemical vapour deposition (MOCVD) or molecular beam epitaxy (MBE) deposition processes required to make such crystalline, lattice matched multijunction solar cells are prohibitively costly for manufacture and deployment of such solar cells in terrestrial applications.

Semiconductors such as perovskites, with continuously tunable bandgaps and the promise of lower cost manufacturing techniques thus offer great opportunities to meet the performances of III-V multijunction tandem solar cells at costs suitable for terrestrial PV deployment. Their bandgap can be tuned between $\sim 1.2$ and $2.3 \mathrm{eV}$ by adjusting the composition of the A cation ( $\mathrm{Cs}$, methylammonium and formamidinium and mixtures thereof), $\mathrm{B}$ metal $(\mathrm{Pb}$ to $\mathrm{Sn}$ and mixtures thereof), and $\mathrm{X}$ halide (I to $\mathrm{Br}$ ) within the $\mathrm{ABX}_{3}$ crystal structure ${ }^{4,5}$. Several research groups have made both monolithically integrated $(2 \mathrm{~T})^{6-8}$ and mechanically stacked $(4 \mathrm{~T})^{9-11}$ perovskite-Si double junction tandems, reaching impressive $26.4 \% 4 \mathrm{~T}$ and $23.6 \% 2 \mathrm{~T}$ tandem efficiencies. We recently also made all-perovskite double junction tandem solar cells with $20.3 \%$ efficiency in a $4 \mathrm{~T}$ and $17.0 \%$ efficiency in a $2 \mathrm{~T}$ architecture $^{12}$, while Yan et al obtained a $21 \%$ efficient all-perovskite $4 \mathrm{~T} \operatorname{tandem}^{13}$.

Building an efficient multi-junction solar cell composed of many thin film layers is not trivial; care must be taken to minimize parasitic absorption and thin film interference effects to maximise and match solar light absorption in each absorber layer, whilst also allowing as close 
to perfect as possible photovoltaic diode operation. However, before embarking on such a mammoth research challenge, it would be very useful to know how much of an efficiency (and energy yield) gain is feasibly achievable by moving towards a two or even three junction stack. In addition, knowing the specific material requirements, such as ideal bandgaps of absorber layers and optical properties of charge collection and recombination layers, would save a lot of time and effort in an otherwise trial and error approach to research. In this work, we couple an optical model with a simple device model in order to optimise and evaluate the performances and energy yields achievable for monolithically integrated all perovskite multijunction (double and triple) and perovskite-Si triple junction solar cells. We base our simulations on experimentally derived $\mathrm{n}$ and $\mathrm{k}$ values for the different layers (listed in the supporting information in Tables S1,2,3), and extract parameters for our simulated current-voltage (JV) curves from experimentally measured JV curves of relevant perovskite (and $\mathrm{Si}$ ) solar cells. The simulation is effectively a detailed balance calculation using experimentally derived absorption spectra, ideality factor, radiative efficiency, and shunt and series resistances. We find that with current device architectures, it is possible to obtain a $31.9 \%$ efficient $2 \mathrm{~T}$ all perovskite tandem cell (2PJ), a $33.1 \%$ all perovskite triple junction cell (3PJ), and a $35.3 \%$ efficient perovskiteSi triple junction cell (2PSJ) under AM1.5G conditions. We also evaluated "idealized" tandem architectures, by making certain assumptions about yet-to-be-realised charge extraction and charge recombination layers, and determine that future work feasibly yields $33.8 \%, 36.6 \%$, and $38.8 \%$ for $2 \mathrm{PJ}, 3 \mathrm{PJ}$ and $2 \mathrm{PSJ}$ performances respectively, assuming that the optoelectronic quality of all perovskite junctions matches the current best-in-class single junction perovskite solar cells. Crucially, these devices can all maintain their high performances under varying conditions to deliver high annual energy yields, which we calculated for the Mohave desert (a preferable location for utility scale solar power plants) and Seattle (a heavily overcast region), and find negligible energy losses due to spectral mismatch. 
We note, that we have only considered monolithically integrated $2 \mathrm{~T}$ multi-junction cell architectures in our work, and not multi-terminal, physically stacked architectures. It may in principle be possible to obtain both higher efficiency and increased energy yield from a physically stacked multi-junction cell, if optical losses related to charge extraction are not considered $^{14-16}$. However, every additional junction will introduce optical losses due to the requirement to extract current at every inter-cell electrode, which will likely to induce at least $10 \%$ drop in current in every subsequent junction and hence be detrimental to practical efficiency. In particular, the additional TCOs and module gridlines will introduce additional optical losses and increase the cost of the modules.

We have calculated the Shockley-Queisser limiting efficiency for ideal tandem and triple junction cells, and show this in the supplementary information Figure S1. Although we find that maximum efficiencies of triple junction cells under AM1.5 $100 \mathrm{mWcm}^{-2}$ sun light could surpass $46 \%$, no real solar cell can achieve such efficiency. The actual expected power conversion efficiency (PCE) is significantly lower than the Shockley-Queisser limit because real solar cells suffer from optical losses such as parasitic absorption, reflection losses and nonrectangular absorption profiles at the band edge, in addition to electrical losses such as series resistance, low shunt-resistances leading to high leakage current, and non-radiative recombination. To account for these factors in our simulations, we used a transfer matrix (TMM) and device modelling approach, previously described by Hörantner and Snaith ${ }^{15}$ to simulate a more realistic expectation of PCE in experimentally realisable multijunction device stacks. Here, we theoretically shift the bandgaps of the perovskite layers and then optimise the thicknesses of all layers to arrive at the highest achievable efficiency. We use $\mathrm{n}$ and $\mathrm{k}$ values obtained by ellipsometry measurements for $\mathrm{CH}_{3} \mathrm{NH}_{3} \mathrm{PbI}_{3}$ by Loper et al. ${ }^{17}$ for the wider bandgap lead based cells, and then apply the Kramers-Kronig relation to obtain $\mathrm{n}$ and $\mathrm{k}$ values 
for a wide range in top cell $\left(\mathrm{E}_{\mathrm{g}}>1.4 \mathrm{eV}\right)$ bandgaps. For the low bandgap perovskites $\left(\mathrm{E}_{\mathrm{g}}<1.4\right.$ $\mathrm{eV}$ ) we use measured $\mathrm{n}$ and $\mathrm{k}$ values, which we determined from our ellipsometry measurements of a $\mathrm{MA}_{0.4} \mathrm{FA}_{0.6} \mathrm{Sn}_{0.6} \mathrm{~Pb}_{0.4} \mathrm{I}_{3}$ film, a composition used in the best cells of this class by Yan et al. ${ }^{13}$ (see Figures S2 and S3a). We fit the best experimentally obtained J-V curves of low bandgap perovskites, high bandgap perovskites, and silicon heterojunction solar cells to a non-ideal diode model to extract ideality factor, radiative efficiency and series and shunt resistances (see Figure S3b). We note that it is crucial that tandem sub-cells have low ideality factors and high shunt resistances to prevent them from performing less efficiently at lower intensities. Correa-Baena et al. ${ }^{18}$ have recently shown that the ideality factor within perovskite solar cells can approach 1 in typical operation.

We illustrate the device architectures for which we carried out our simulations in Figure 1a. We assume a perovskite-on-perovskite tandem device architecture similar to the one experimentally demonstrated by Eperon and Leijtens et al. ${ }^{12}$, with bandgaps of $0.9-1.35 \mathrm{eV}$ and 1.5-2 $\mathrm{eV}$ for the bottom and top perovskite absorber layers respectively. The simulated allperovskite triple junction architecture is based on a similar architecture as the double junction architecture with an additional top perovskite absorber of wider bandgap from $1.9-2.3 \mathrm{eV}$. The middle perovskite absorber ranges from $1.5-1.9 \mathrm{eV}$ and we keep the bottom perovskite absorber fixed at $1.22 \mathrm{eV}$, which is currently the lowest bandgap reported for an efficient perovskite solar cell ${ }^{13,19}$. For the triple junction perovskite-on-perovskite-on-silicon architecture we include a silicon heterojunction (SHJ) bottom cell, as we have used previously for perovskite-on-silicon double junction simulations ${ }^{15}$. We simulate the middle and top perovskite absorbers with varied bandgaps of $1.35-1.75 \mathrm{eV}$ and $1.8-2.2 \mathrm{eV}$ respectively. Both, the middle and top perovskite absorber layers are sandwiched between the typical organic charge extraction layers, 2,2',7,7'-Tetrakis-(N,N-di-4-methoxyphenylamino)-9,9'spirobifluorene (Spiro-OMeTAD) and $\mathrm{C}_{60}$ as the hole-transport material (HTM) and the 
electron-transport material (ETL) respectively. We constrain all the layer thicknesses to be within a range which we determine to be experimentally feasible based on current state-of-theart devices, which we state within the illustration of Figure 1a and in Tables S1,2,3.

We show the results for double and triple-junction perovskite solar cells by plotting them as contour plots in Figure 1b,c,d. Encouragingly, we find that perovskite-on-perovskite tandem solar cells can achieve efficiencies of over $32 \%$ when employed in bandgap combinations of $1.15 \mathrm{eV}$ and $1.75 \mathrm{eV}$, which is a very similar efficiency as achievable with perovskite-on-silicon hybrid tandem cells. However, if we limit the band gap of the perovskite rear cell to $1.22 \mathrm{eV}$, this tandem efficiency drops back to $31.9 \%$. This can be more than recuperated however by moving to the all-perovskite triple junction, for which we determine a peak efficiency of $33.1 \%$, when assuming a $1.22 \mathrm{eV}$ bottom cell. Even more encouraging, we determine that a perovskite double junction on top of a silicon heterojunction (SHJ) solar cell can achieve $35.3 \%$ efficiency, when engineered with perovskite bandgaps of $1.44 \mathrm{eV}$ and 1.95 $\mathrm{eV}$, which would beat the current record efficiency for III-VI triple junction solar cells deposited via epitaxial growth ${ }^{2}$. We note that each individual junction we have simulated from within the triple junction stacks are approximately $~ 21-22 \%$ efficient, so the tandems offer significant improvements over the single junction solar cell efficiency. 

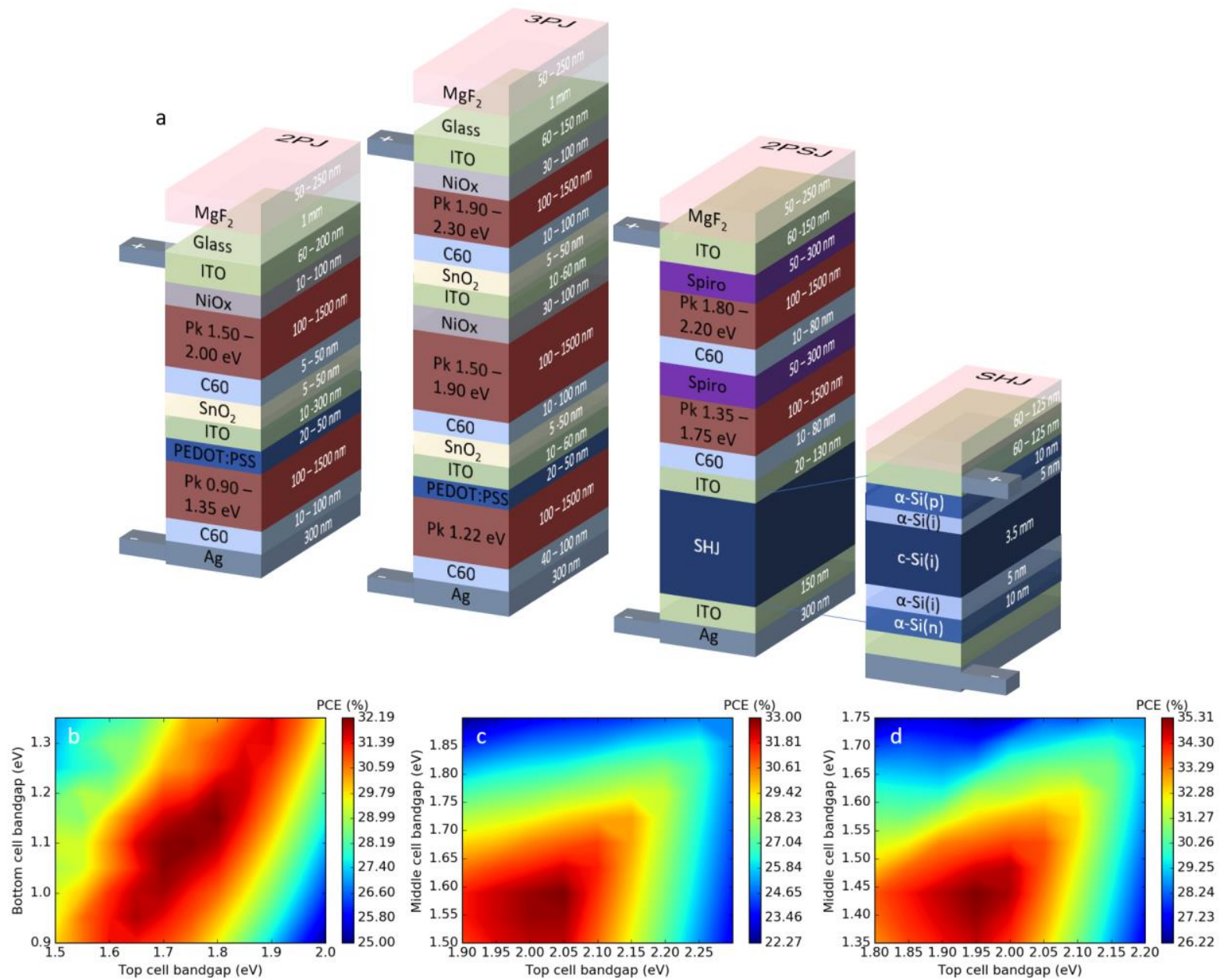

Figure 1: a) Illustration of the perovskite tandem junction, all perovskite triple junction and perovskiteon-silicon triple junction device architectures. The thickness range used in the optical model for each layer is written upon the illustration and detailed in Tables S1,2,3. b) All-perovskite double junction PCE for varied band-gap combinations of bottom and top absorber. c) All-perovskite triple junction solar cell PCE for varied band-gap combinations of bottom and top absorber on top of perovskite base absorber with fixed $1.22 \mathrm{eV}$ band-gap. d) Perovskite-perovskite-silicon triple junction solar cell PCE for varied band-gap combinations of bottom and top perovskite absorber on top of fixed band-gap SHJ base absorber.

In order to understand where losses are occurring, and where further improvements may be realised, we show our calculated external quantum efficiencies (EQEs) and J-V curves for the optimised multi-junction devices in Figure 2. Though the maximum PCE in a $2 \mathrm{PJ}$ is obtained with a $1.15 \mathrm{eV}$ bottom cell, we chose to plot the EQE and JV curve of the 2PJ using 
an already achieved $1.22 \mathrm{eV}$ bandgap for the bottom cell. We note that we do not determine perfect current matching between the front and rear cells in our calculated JV curves. This is due to the finite step size in our parameter optimisation. However, a slight mismatch in shortcircuit current leads to a slight improvement in FF of the tandem cell, which predominantly compensates on overall efficiency, which is what our calculation has maximised. Although the $1.22 \mathrm{eV}$ bottom cell bandgap is not ideal, we demonstrate that it can still yield high tandem performances of $\sim 31.5 \%$ when paired with a $1.83 \mathrm{eV}$ top cell. We show all the optimised layer thickness in the supplementary information in Tables $\mathrm{S} 1,2,3$. We reach the ideal tandem performances with very thick bottom absorber thicknesses $(1.5 \mu \mathrm{m})$. While recent work has demonstrated the feasibility of making efficient $1 \mu \mathrm{m}$ thick $\mathrm{MA}_{0.4} \mathrm{FA}_{0.6} \mathrm{Sn}_{0.6} \mathrm{~Pb}_{0.4} \mathrm{I}_{3}$ solar cells ${ }^{13}$, we also evaluated the effect of limiting the bottom absorber thickness, and plot the obtainable PCEs 2PJs as a function of $1.22 \mathrm{eV}$ bottom cell thickness in Figure S4a. We find that even at only $500 \mathrm{~nm}$ thickness in the bottom cell, we obtain a $31 \%$ tandem if the top cell bandgap is raised to $1.86 \mathrm{eV}$; the lower photocurrent is somewhat offset by an increase in voltage, as we see in the modelled JV curves in Figure S4b. As a result, the thickness dependence of perovskite tandems is not that steep, provided that top cells with high output voltages can be realized.

The all-perovskite triple junction device for which we show the EQE and the JV curves in Figure $2 \mathrm{~b}$ and $2 \mathrm{e}$ has a relatively low matched current around $10 \mathrm{~mA} / \mathrm{cm}^{2}$, mainly because interference effects lead to large fringes in the bottom absorber. However, when we analyse the EQE and JV curves of the triple junction perovskite-on-silicon device in Figure $2 \mathrm{c}$ and $2 \mathrm{f}$, we find that fewer fringes occur in the EQE and that the currents match at higher currents around $13 \mathrm{~mA} / \mathrm{cm}^{2}$. This is mainly due to the large thickness of the Si bottom cell $(100 \mathrm{~s} \mu \mathrm{m})$, limiting thin film interference effects. We also point out that to perform the TMM, we assume that all light within most of the layers within the stacks is coherent. Realistic solar cells will have some roughness simply due to the fact that they are polycrystalline. The results also 
suggest that designing textured light trapping architectures such as those used in most commercial silicon solar cells to minimize reflection losses could lead to very large improvements in tandem performances, with the biggest gains to be obtained for triple junction all-perovskite tandems.
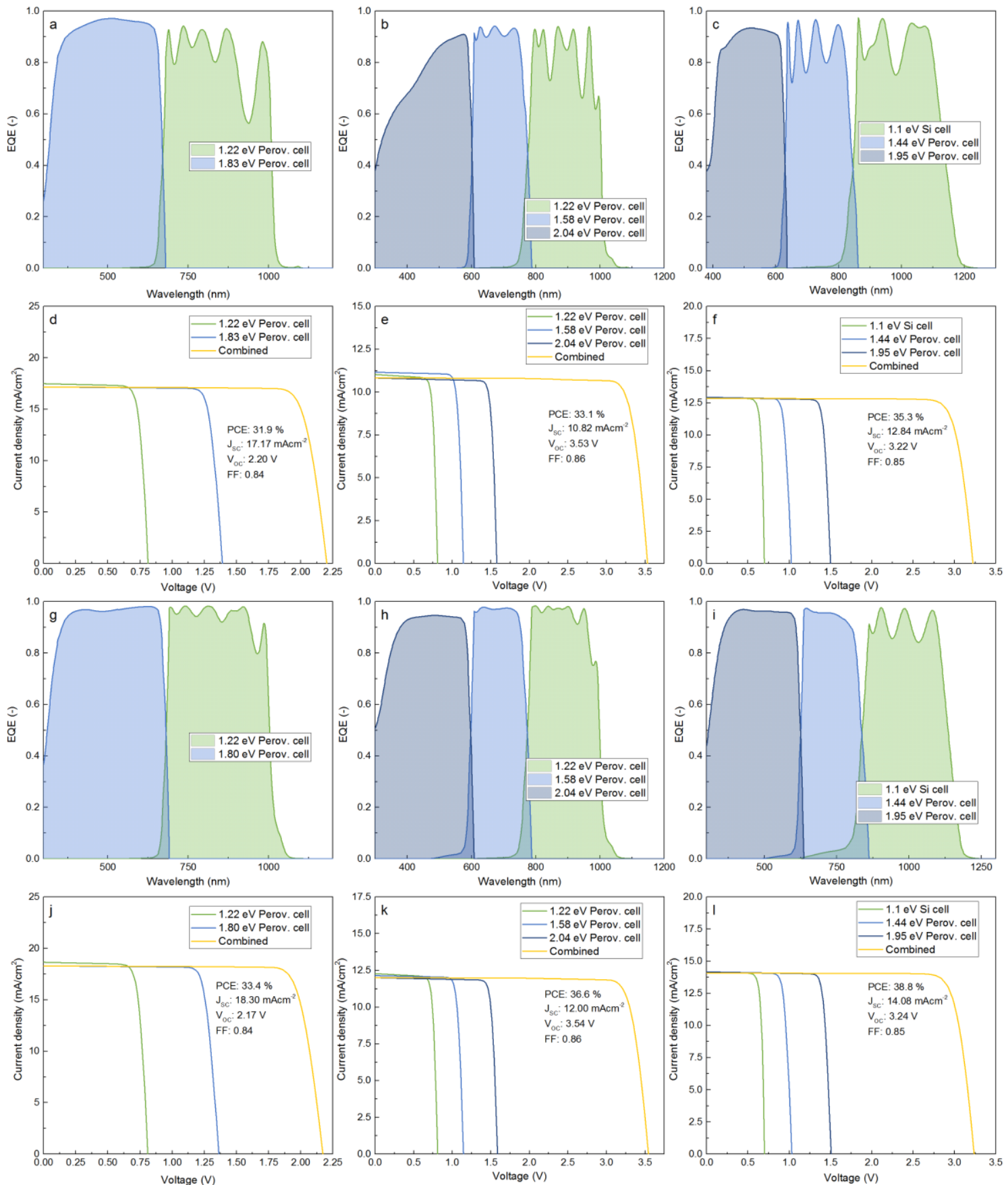
Figure 2: Calculated EQEs and resulting JV curves of optimised all-perovskite double junction (a,d), all-perovskite triple junction (b,e) and perovskite-perovskite-silicon (c,f) solar cells with ideal bandgap combination and currently achievable thickness constraints using optical transfer matrix and singlediode device modelling. Respective calculations of optimised all-perovskite double junction (g,j), allperovskite triple junction $(\mathrm{h}, \mathrm{k})$ and perovskite-perovskite-silicon (i,l) solar cells with ideal bandgap combination and optically idealised thickness constraints.

We base the results we present above on proven device architectures using readily achievable thicknesses for all the layers in the device stacks. However, we find that the resultant EQEs exhibit strong interference fringes, which significantly limit photocurrent generation in the rear perovskite cells. To evaluate the primary sources of such interference, and hence give an indication of the future direction for optimising the device stacks, we varied some of the constraints we placed on the device architecture, specifically for material layers which we found to introduce optical losses. In short, we find that the largest improvements come from thinning all non-perovskite charge extraction and recombination layers to $5 \mathrm{~nm}$, and by removing the PEDOT:PSS, which is optically lossy. We make smaller improvements by removing the ITO recombination layer, which may be feasible when making vapor deposited tandem solar cells using thin, doped recombination layers, such as those already realised by Bolink and co workers ${ }^{20}$. We note that it is essential not to introduce any additional electrical losses to the multijunction solar cell performance associated with the recombination layer. However, existing solutions already appear to enable relatively low-loss recombination contacts, largely due to the ease of using varying conductive contacts with perovskites, which do not require lattice matching or special substrates to enable high quality optoelectronic properties. We exemplify the impact of these changes individually in Figures S5 and S6, and implement all these changes into the "ideal" multi-junction stacks for which we show the 
modelled EQE spectrum and JV curves in Figure 2g-1. We optimised such devices with the material stacks and thickness constraints illustrated in Figure S6a. We find that the optically optimised devices can reach $33.8,36.6$, and $38.8 \%$ for 2PJs, 3PJs, and 2PSJs respectively and show the results for varied bandgaps in Figure S7a,b,c. This is significantly beyond any presently realised efficiency for any PV technology, including III-V semiconductor multijunction devices. This therefore gives significant motivation to both develop the range of required band gap single junction perovskite cells with comparably high efficiency and to integrate them into device stacks with optically thin charge collection and recombination layers.

For the simulations, we have varied the bandgaps to find the absolute highest achievable PCEs. However, the ideal bandgap combinations may not always use the most stable perovskite compositions; small bandgap perovskite solar cells incorporating Sn on the B site suffer from degradation in air, since $\mathrm{Sn}^{2+}$ species are oxidized to $\mathrm{Sn}^{4+21-23}$. Furthermore, wide bandgap perovskite solar cells using high $\mathrm{Br}$ contents are prone to undergoing halide segregation (referred to as the Hoke effect) ${ }^{24,25}$. In the case of $2 \mathrm{PJ}$, one could reduce the Sn content of the low bandgap absorber to make it more air stable, increasing the bandgap. Similarly, one could constrain the Br content by limiting the top cell bandgap below $1.75 \mathrm{eV}$, which has been demonstrated to yield efficient and stable solar cells ${ }^{26}$. If we limit ourselves to using only $25 \%$ Sn, which has been shown to yield much improved air stability ${ }^{27}$ at a $1.35 \mathrm{eV}$ bandgap, and pair this with a $\sim 1.75 \mathrm{eV}$ bandgap top cell, we estimate that we could surprisingly still obtain a $\sim 30.5 \%$ tandem provided all the layers are optimized to achieve current matching. This approach relies on the ability to tune the absorber thicknesses; in particular, the bottom absorber must be able to approach $\sim 1 \mu \mathrm{m}$ to enable reasonably high current matching. This strategy is unlikely to work for the 3PJs because these rely more heavily on having a small enough bottom absorber bandgap. 
Similarly, the optimal $1.44 \mathrm{eV}$ perovskite middle absorber for the silicon based triple junction architecture has not been shown in a well performing device and would require the use of mixed $\mathrm{Sn}-\mathrm{Pb}$ perovskites or the phase-unstable $\mathrm{FAPbI}_{3}^{28,29}$ in the middle cell, possibly limiting the lifetime of the Si bottom cell. However, if we limit ourselves to the band gap of the current world record perovskite cell at $1.50 \mathrm{eV}^{1}$, we calculate that a triple junction perovskite-perovskite-silicon device will deliver a reduced PCE of $34.6 \%$ when comprised of the bandgap combination $1.50 \mathrm{eV}$ and $2.0 \mathrm{eV}$ because the perovskite absorbers cannot match the high current from the SHJ. We show the EQEs and JVs of these calculations for currently feasible and optically ideal stacks in Figure S8. Since we have already predicted a two junction perovskite-Si tandem to yield $32 \%$ PCE, the success of the 2PSJ relies heavily on the continued development of stable and efficient perovskites in the $\sim 1.4-1.5 \mathrm{eV}$ bandgap region. An even greater challenge is the requirement for an efficient and stable $\sim 2 \mathrm{eV}$ top cell, which has not been reported to date. Thus far, increasing the bandgap past $\sim 1.75 \mathrm{eV}$ has not resulted in the expected increases in $\mathrm{V}_{\mathrm{OC}}$, and the high bromide contents of compounds with these bandgaps makes them relatively unstable.

Measuring or estimating the efficiency of a solar cell under a standardised light source (AM1.5 $100 \mathrm{mWcm}^{-2}$ irradiance) is simply a means of determining a parameter which should reflect the electrical energy yield the device would deliver if deployed under real world solar illumination. However, due to spectral variations in terrestrial sun light induced from changes in atmospheric conditions and time of day variations, the absolute energy yield of a solar cell can vary. Specifically for multi-junction solar cells, the requirement for current matching between sub cells adds an additional complexity to energy yield estimations. In order to assess if the increased multi-junction cell efficiency can translate into increased energy yield, we carry out energy yield (EY) simulations for real world conditions in the Mohave desert and Seattle, similar to that previously described by Hörantner and Snaith ${ }^{15}$. We note that we do not account 
for temperature coefficient of the cells, which will generally reduce the efficiency of a solar junction with increased temperature. However, assuming the temperature coefficient is similar for all junctions, this will not greatly affect the relative changes in energy yield ${ }^{30}$, although this does result in us slightly overestimating the absolute energy yields for each configuration. We present the results for tandem devices with a fixed tilting angle $\left(34.9^{\circ}\right)$ in Figure 3 and find that the EY is maximized at similar bandgap combinations to where the PCE was optimized at AM1.5G. Importantly, we find that the energy yields for the monolithically integrated tandems are appropriately higher than that of either a single junction perovskite solar cell or the single junction SHJ modelled previously at these conditions; the tandems do not suffer from significant additional losses due to current matching issues. We note that we have only considered absolute efficiency and energy yield as factors to assess the feasibility of developing multi-junction perovskite solar cells. We have not considered the impact this may have on the scaling to large area and achieving a high yield in a future solar cell manufacturing facility. Clearly, extremely good deposition control over the uniformity (physical and optoelectronic) of every layer will be required due to the multiple layers present in the multi-junction cells: Any given process will have a yield for producing an individual layer within spec, and every additional layer in the cell compounds the yield of the final manufactured cells and modules. However, in favour of perovskites with respect to III-V multi-junction cells, lattice matching between subsequent layers is not required.

To evaluate the energy yield in comparison to the corresponding AM1.5 PCEs, we calculate the "capacity factors" by dividing our calculated energy yield by the peak power at $\mathrm{AM} 1.5 / 0^{\circ}$, where the $0^{\circ}$ stands for perpendicular angle of incidence of the light. We tabulate the values in Table 1, and compare the multi-junction cells to the values from single junction silicon SHJ and single junction perovskite cell of bandgap $1.65 \mathrm{eV}^{15}$. Remarkably, we find that the capacity factor of the $2 \mathrm{PJ}$ device outperforms that of the single junction silicon cell with 
$2.17 \mathrm{kWh} / \mathrm{W}_{\mathrm{p}}$ vs $2.16 \mathrm{kWh} / \mathrm{W}_{\mathrm{p}}$. Even more surprisingly, we estimate that the triple junction cells only perform slightly worse, with the $3 \mathrm{PJ}$ (the architecture with the lowest capacity factor) cell still delivering $2.14 \mathrm{kWh} / \mathrm{W}_{\mathrm{p}}$. Therefore, all multijunction cells are within an absolute range of $+/-0.02 \mathrm{kWh} / \mathrm{W}_{\mathrm{p}}$ of the single junction silicon cell, and all deliver significantly increased absolute energy yield. Moving to the "ideal" multijunction stacks, we estimate that the capacity factors of $2 \mathrm{PJ}$ and $2 \mathrm{PSJ}$ devices can be even further improved to $2.17 \mathrm{kWh} / \mathrm{W}_{\mathrm{p}}$ and $2.17 \mathrm{kWh} / \mathrm{W}_{\mathrm{p}}$, as we show in Table 1 . In the best scenario of the $2 \mathrm{PSJ}$, we estimate a relative increase in energy yield of $73 \%$ with respect to the single junction silicon heterojunction cell. We note that the diffuse irradiance spectrum of Mohave desert is significantly blue shifted compared to the AM1.5 spectrum, coming from Raleigh scattering, which is the reason why the untracked single junction perovskite cell yields a substantially higher capacity factor than the SHJ single junction device ${ }^{15}$.

For comparison with a more overcast region, we additionally carried out EY simulations for deployment in Seattle at a fixed tilt angle of $47.5^{\circ}$. We find that the relative energy yield gain is slightly reduced in a cloudier region. For instance, the capacity factor of single junction perovskite is $1.07 \mathrm{kWh} / \mathrm{kW}_{\mathrm{p}}$ for a single junction perovskite, 1.06 for a single junction $\mathrm{SHJ} \mathrm{kWh} / \mathrm{kW}_{\mathrm{p}}, 1.05 \mathrm{kWh} / \mathrm{kW}_{\mathrm{p}}$ for a $2 \mathrm{PJ}$ device, $1.02 \mathrm{kWh} / \mathrm{kW}_{\mathrm{p}}$ for a $3 \mathrm{PJ}$ device and $1.03 \mathrm{kWh} / \mathrm{kW}_{\mathrm{p}}$ for a $2 \mathrm{PSJ}$ device. The decrease of capacity factors can be assigned to the fact that weather conditions change more often in Seattle, leading to a more frequent mismatch in currents. Absolute energy yield does still significantly increase with the added junctions, but probably, assuming costs of manufacturing and manufacturing yields would be influenced, triple junction solar cells would be less attractive than tandems in locations like Seattle. However, tandems still significantly outperform single junctions. 

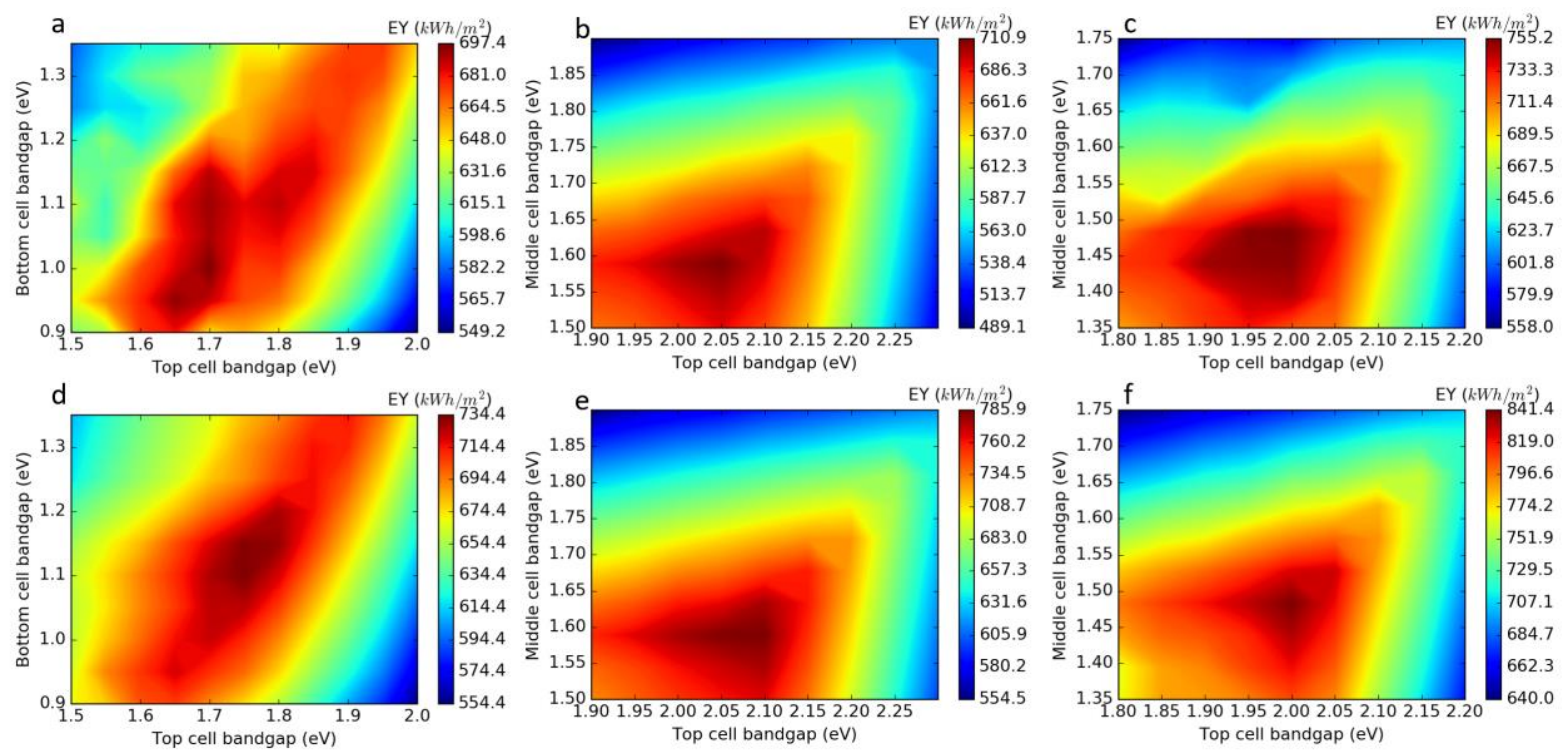

Figure 3: All-perovskite double junction energy yield in Mohave desert for varied band-gap combinations of bottom and top absorber with currently feasible thickness constraints (a) and with optically idealised thickness constraints (d). All-perovskite triple junction solar cell energy yield in Mohave desert for varied band-gap combinations of bottom and top absorber on top of fixed perovskite base absorber $\left(\mathrm{E}_{\mathrm{g}}=1.22 \mathrm{eV}\right)$ with currently feasible thickness constraints (b) and with optically idealised thickness constraints (e). Perovskite-perovskite-silicon triple junction solar cell energy yield in Mohave desert for varied band-gap combinations of bottom and top perovskite absorber on top of fixed band-gap SHJ base absorber with currently feasible thickness constraints (c) and with optically idealised thickness constraints (f).

Table 1: Tabulated optimised PCE with corresponding annual energy yield, relative energy yield gain and capacity factor at Mohave desert and Seattle for single junction devices and tandem stacks with currently feasible thickness constraints and with optically idealised thickness constraints.

\begin{tabular}{|l|c|c|c|c|c|c|c|}
\hline Stack: & $\begin{array}{c}\text { PCE } \\
(\%)\end{array}$ & $\begin{array}{c}\text { EY } \\
\left(\mathrm{kWh} / \mathrm{m}^{2}\right)\end{array}$ & $\begin{array}{c}\text { EY gain } \\
\text { on SHJ } \\
\text { cell }(\%)\end{array}$ & $\begin{array}{c}\text { Capacity } \\
\text { factor } \\
\left(\mathrm{kWh} / \mathrm{W}_{\mathrm{p}}\right)\end{array}$ & $\begin{array}{c}\mathrm{EY} \\
\left(\mathrm{kWh} / \mathrm{m}^{2}\right)\end{array}$ & $\begin{array}{c}\text { EY gain } \\
\text { on SHJ } \\
\text { cell }(\%)\end{array}$ & $\begin{array}{c}\text { Capacity } \\
\text { factor } \\
\left(\mathrm{kWh} / \mathrm{W}_{\mathrm{p}}\right)\end{array}$ \\
\hline & \multicolumn{3}{|c|}{ Mohave desert } & \multicolumn{3}{|c|}{ Seattle } \\
\hline
\end{tabular}




\begin{tabular}{|r|c|c|c|c|c|c|c|}
\hline $\begin{array}{r}\text { Perovskite } \\
\text { single }^{15}\end{array}$ & 21.96 & 489.33 & 0.3 & 2.23 & 233.94 & -1.8 & 1.07 \\
\hline SHJ single $^{15}$ & 22.58 & 487.65 & 0.0 & 2.16 & 238.28 & 0.0 & 1.06 \\
\hline 2PJ & 32.19 & 697.40 & 43.0 & 2.17 & 337.53 & 41.7 & 1.05 \\
\hline 3PJ & 33.00 & 710.90 & 45.8 & 2.15 & 336.6 & 41.3 & 1.02 \\
\hline 2PSJ & 35.31 & 755.20 & 54.9 & 2.14 & 364.2 & 52.8 & 1.03 \\
\hline Ideal 2PJ & 33.80 & 734.40 & 50.6 & 2.17 & 353.35 & 48.3 & 1.05 \\
\hline Ideal 3PJ & 36.55 & 785.90 & 61.2 & 2.15 & 371.3 & 55.8 & 1.02 \\
\hline Ideal 2PSJ & 38.80 & 841.40 & 72.5 & 2.17 & 399.6 & 67.7 & 1.03 \\
\hline
\end{tabular}

We demonstrate that high efficiency perovskite tandem solar cells are possible with currently available material sets, provided they are carefully integrated into a tandem stack. Assuming optimisation of already demonstrated device architectures, we estimate that AM1.5 solar cell efficiencies close to $32 \%$ are feasible in all perovskite tandem cells with the current generation of $\mathrm{Sn}-\mathrm{Pb}$ low bandgap bottom absorbers. This suggests that we can expect even higher efficiencies in the future as the voltage deficits and parasitic resistances continue to improve. We also demonstrate that triple junction cells, composed of either all perovskite junctions or two perovskite junctions on silicon could deliver potential performances to above 33 and $35 \%$ respectively. Through analysis of the material stacks, we have identified key parameters responsible for optical losses, and in an improved device architecture we estimate feasible power conversion efficiencies of $33.8,36.6$, and $38.8 \%$ for all-perovskite tandem (2PJ), all perovskite triple (3PJ) and perovskite tandem in silicon (2PSJ) cells. Even more encouragingly, we have calculated the energy yield of all our optimised multijunction cells using real world irradiance data, and find that they deliver a comparative boost in energy yield, with our most optimistic idealised structure delivering a $73 \%$ increase in energy yield, as compared to a single junction silicon cell.

In order to realise the highest efficiencies for the device stacks we have considered here, a number of factors arise as key areas which require targeted development. 
i) For the all-perovskite tandem cell, we must realise a 1.8 to $1.9 \mathrm{eV}$ perovskite cell with a $\mathrm{V}_{\mathrm{OC}}$ of 1.35 to $1.4 \mathrm{~V}$.

ii) For the all perovskite tandem and the all perovskite triple junction, we must realise thick (order 1 micron) low band gap perovskite cells, incorporating Sn, which are stable enough to survive environmental aging.

iii) For all triple junction architectures, we must realise a wide band gap $(\sim 2.0 \mathrm{eV})$ perovskite solar cell with an open-circuit voltage $>1.5 \mathrm{~V}$.

iv) To minimise optical losses, we must develop routes to deposit the multilayers without destroying the underlying materials, and with continuous charge extraction layers of minimal thickness (on the order of $5 \mathrm{~nm}$ ).

Our JV characteristics are derived from the present best-in-class JV curves in the perovskite literature and so further reductions in the loss-in-potential and non-radiative recombination in perovskite solar cells will drive the performances of the multi-junction cells beyond the numbers we have determined here. Additionally, we have only considered coherent light in all our calculations, and have not introduced any advanced strategies for enhancing light scattering within the absorber layers, which could further increase the photocurrent generation. We therefore consider our estimations as a "conservative" view of the future prospects for perovskite multi-junction solar cells, and our results demonstrate that all-perovskite multijunction solar cells are likely to deliver an ultimately low cost solar technology with efficiencies beating even the most advanced classical semiconductor devices. 


\section{Acknowledgements}

M.T.H was supported by EPSRC EP/M024881/1 and Oxford Photovoltaics Inc. T.L. is funded by a Marie Sklodowska Curie International Fellowship under grant agreement H2O2IF-GA2015-659225. M.E.Z. acknowledges support from DOE BES DE-SC0013957. G.E.E. is supported by the European Union's Framework Programme for Research and Innovation Horizon 2020 (2014-2020) under the Marie Skłodowska-Curie Grant Agreement No. 699935. Part of this work was conducted at the Molecular Analysis Facility, a National Nanotechnology Coordinated Infrastructure site at the University of Washington, which is supported in part by the National Science Foundation (Grant ECC-1542101), the University of Washington, the Molecular Engineering \& Sciences Institute, the Clean Energy Institute, and the National Institutes of Health. M.G.C. has received funding from the European Research Council (ERC) under the European Union's Horizon 2020 research and innovation programme (Marie Skłodowska-Curie Individual Fellowships, IF-EF, grant agreement no 659667).

\section{Supporting Information}

Supporting Information in form of a pdf document and a text based datafile is available on the ACS Publications website. The pdf document contains a detailed description of the methods, the supporting figures as well as supporting tables. The datafile contains the complex refractive index data of the narrow bandgap $\mathrm{MA}_{0.4} \mathrm{FA}_{0.6} \mathrm{Sn}_{0.6} \mathrm{~Pb}_{0.4} \mathrm{I}_{3}$ material.

\section{References}

(1) Yang, W. S.; Park, B.-W.; Jung, E. H.; Jeon, N. J.; Kim, Y. C.; Lee, D. U.; Shin, S. S.; Seo, J.; Kim, E. K.; Noh, J. H.; et al. Iodide Management in Formamidinium-LeadHalide-based Perovskite Layers for Efficient Solar Cells. Science 2017, 356, 1376 LP1379. 
(2) Green, M. A.; Hishikawa, Y.; Warta, W.; Dunlop, E. D.; Levi, D. H.; Hohl-Ebinger, J.; Ho-Baillie, A. W. H. Solar Cell Efficiency Tables (Version 50). Prog. Photovoltaics Res. Appl. 2017, 25, 668-676.

(3) Yoshikawa, K.; Kawasaki, H.; Yoshida, W.; Irie, T.; Konishi, K.; Nakano, K.; Uto, T.; Adachi, D.; Kanematsu, M.; Uzu, H.; et al. Silicon Heterojunction Solar Cell with Interdigitated Back Contacts for a Photoconversion Efficiency over 26\%. Nat. Energy 2017, 2, 17032.

(4) Noh, J. H.; Im, S. H.; Heo, J. H.; Mandal, T. N.; Seok, S. Il. Chemical Management for Colorful, E Ffi Cient, and Stable Inorganic - Organic Hybrid Nanostructured Solar Cells. 2013, 2, 28-31.

(5) Hao, F.; Stoumpos, C. C.; Chang, R. P. H.; Kanatzidis, M. G. Anomalous Band Gap Behavior in Mixed $\mathrm{Sn}$ and $\mathrm{Pb}$ Perovskites Enables Broadening of Absorption Spectrum in Solar Cells. J. Am. Chem. Soc. 2014, 136, 8094-8099.

(6) Albrecht, S.; Saliba, M.; Correa Baena, J. P.; Lang, F.; Kegelmann, L.; Mews, M.; Steier, L.; Abate, A.; Rappich, J.; Korte, L.; et al. Monolithic Perovskite/siliconHeterojunction Tandem Solar Cells Processed at Low Temperature. Energy Environ. Sci. 2016, 9, 81-88.

(7) Werner, J.; Weng, C. H.; Walter, A.; Fesquet, L.; Seif, J. P.; De Wolf, S.; Niesen, B.; Ballif, C. Efficient Monolithic Perovskite/Silicon Tandem Solar Cell with Cell Area $>1$ cm2. J. Phys. Chem. Lett. 2016, 7, 161-166.

(8) Bush, K. A.; Palmstrom, A. F.; Yu, Z. J.; Boccard, M.; Cheacharoen, R.; Mailoa, J. P.; McMeekin, D. P.; Hoye, R. L. Z.; Bailie, C. D.; Leijtens, T.; et al. 23.6\%-Efficient Monolithic Perovskite/silicon Tandem Solar Cells with Improved Stability. Nat. Energy 2017, 2, 17009.

(9) Werner, J.; Barraud, L.; Walter, A.; Bräuninger, M.; Sahli, F.; Sacchetto, D.; Tétreault, N.; Paviet-Salomon, B.; Moon, S.; Allebé, C.; et al. Efficient Near-InfraredTransparent Perovskite Solar Cells Enabling Direct Comparison of 4-Terminal and Monolithic Perovskite/Silicon Tandem Cells. ACS Energy Lett. 2016, 1, 474-480.

(10) Duong, T.; Wu, Y.; Shen, H.; Peng, J.; Fu, X.; Jacobs, D.; Wang, E.; Kho, T. C.; Fong, K. C.; Stocks, M.; et al. Rubidium Multication Perovskite with Optimized Bandgap for Perovskite-Silicon Tandem with over 26\% Efficiency. Adv. Energy Mater. 2017, 1700228, 1-11.

(11) Bailie, C. D.; Christoforo, M. G.; Mailoa, J. P.; Bowring, A. R.; Unger, E. L.; Nguyen, W. H.; Burschka, J.; Pellet, N.; Lee, J. Z.; Grätzel, M.; et al. Semi-Transparent Perovskite Solar Cells for Tandems with Silicon and CIGS. Energy Environ. Sci. 2014, 8, 956-963.

(12) Eperon, G. E.; Leijtens, T.; Bush, K. A.; Prasanna, R.; Green, T.; Wang, J. T.-W.; McMeekin, D. P.; Volonakis, G.; Milot, R. L.; May, R.; et al. Perovskite-Perovskite Tandem Photovoltaics with Optimized Band Gaps. Science 2016, 354, 861-865.

(13) Zhao, D.; Yu, Y.; Wang, C.; Liao, W.; Shrestha, N.; Grice, C. R.; Cimaroli, A. J.; Guan, L.; Ellingson, R. J.; Zhu, K.; et al. Low-Bandgap Mixed Tin-lead Iodide Perovskite Absorbers with Long Carrier Lifetimes for All-Perovskite Tandem Solar Cells. Nat. Energy 2017, 2, 17018.

(14) Futscher, M. H.; Ehrler, B. Efficiency Limit of Perovskite/Si Tandem Solar Cells. ACS Energy Lett. 2016, 1, 2-7. 
(15) Hörantner, M. T.; Snaith, H. J. Predicting and Optimising the Energy Yield of Perovskite-on-Silicon Tandem Solar Cells under Real World Conditions. Energy Environ. Sci. 2017, 10, 1983-1993.

(16) Futscher, M. H.; Ehrler, B. Modeling the Performance Limitations and Prospects of Perovskite/Si Tandem Solar Cells under Realistic Operating Conditions. ACS Energy Lett. 2017, 2089-2095.

(17) Löper, P.; Stuckelberger, M.; Niesen, B.; Werner, J.; Filipič, M.; Moon, S.-J.; Yum, J.H.; Topič, M.; De Wolf, S.; Ballif, C. Complex Refractive Index Spectra of CH3NH3PbI3 Perovskite Thin Films Determined by Spectroscopic Ellipsometry and Spectrophotometry. J. Phys. Chem. Lett. 2015, 6, 66-71.

(18) Correa-Baena, J.-P.; Turren-Cruz, S.-H.; Tress, W.; Hagfeldt, A.; Aranda, C.; Shooshtari, L.; Bisquert, J.; Guerrero, A. Changes from Bulk to Surface Recombination Mechanisms between Pristine and Cycled Perovskite Solar Cells. ACS Energy Lett. 2017, 2, 681-688.

(19) Prasanna, R.; Gold-Parker, A.; Leijtens, T.; Conings, B.; Babayigit, A.; Boyen, H. G.; Toney, M. F.; McGehee, M. D. Band Gap Tuning via Lattice Contraction and Octahedral Tilting in Perovskite Materials for Photovoltaics. J. Am. Chem. Soc. 2017, 139, 11117-11124.

(20) Forgács, D.; Gil-Escrig, L.; Pérez-Del-Rey, D.; Momblona, C.; Werner, J.; Niesen, B.; Ballif, C.; Sessolo, M.; Bolink, H. J. Efficient Monolithic Perovskite/Perovskite Tandem Solar Cells. Adv. Energy Mater. 2016, 1602121.

(21) Marshall, K. P.; Walker, M.; Walton, R. I.; Hatton, R. A. Enhanced Stability and Efficiency in Hole-Transport-Layer-Free CsSnI3 Perovskite Photovoltaics. Nat. Energy 2016, 1, 16178.

(22) Noel, N. K.; Stranks, S. D.; Abate, A.; Wehrenfennig, C.; Guarnera, S.; Haghighirad, A.-A.; Sadhanala, A.; Eperon, G. E.; Pathak, S. K.; Johnston, M. B.; et al. Lead-Free Organic-Inorganic Tin Halide Perovskites for Photovoltaic Applications. Energy Environ. Sci. 2014, 7, 3061-3068.

(23) Hao, F.; Stoumpos, C. C.; Cao, D. H.; Chang, R. P. H.; Kanatzidis, M. G. Lead-Free Solid-State Organic-Inorganic Halide Perovskite Solar Cells. Nat Phot. 2014, 8, 489494.

(24) Hoke, E. T.; Slotcavage, D. J.; Dohner, E. R.; Bowring, A. R.; Karunadasa, H. I.; McGehee, M. D. Reversible Photo-Induced Trap Formation in Mixed-Halide Hybrid Perovskites for Photovoltaics. Chem. Sci. 2015, 6, 613-617.

(25) Slotcavage, D. J.; Karunadasa, H. I.; McGehee, M. D. Light-Induced Phase Segregation in Halide-Perovskite Absorbers. ACS Energy Lett. 2016, 1199-1205.

(26) McMeekin, D. P.; Sadoughi, G.; Rehman, W.; Eperon, G. E.; Saliba, M.; Horantner, M. T.; Haghighirad, A.; Sakai, N.; Korte, L.; Rech, B.; et al. A Mixed-Cation Lead Mixed-Halide Perovskite Absorber for Tandem Solar Cells. Science 2016, 351, 151155.

(27) Yang, Z.; Rajagopal, A.; Chueh, C. C.; Jo, S. B.; Liu, B.; Zhao, T.; Jen, A. K. Y. Stable Low-Bandgap $\mathrm{Pb}$ ???Sn Binary Perovskites for Tandem Solar Cells. Adv. Mater. 2016, 28, 8990-8997.

(28) Li, Z.; Yang, M.; Park, J.-S.; Wei, S.-H.; Berry, J. J.; Zhu, K. Stabilizing Perovskite Structures by Tuning Tolerance Factor: Formation of Formamidinium and Cesium 
Lead Iodide Solid-State Alloys. Chem. Mater. 2016, 28, 284-292.

(29) Eperon, G. E.; Stranks, S. D.; Menelaou, C.; Johnston, M. B.; Herz, L. M.; Snaith, H. J. Supplementary Information Formamidinium of Formamidinium Lead Trihalide: A Broadly Tunable Perovskite for Efficient Planar Heterojunction Solar Cells. Energy Environ. Sci. 2014, 7, 982.

(30) Duck, B. C.; Dunbar, R. B.; Lee, O.; Anderson, K. F.; Jones, T. W.; Wilson, G. J.; Fell, C. J. Energy Yield Potential of Perovskite-Silicon Tandem Devices. In 2016 IEEE 43rd Photovoltaic Specialists Conference (PVSC); IEEE, 2016; pp. 1624-1629. 